\title{
Exploration On the Residential Needs of Empty Nests and the Adaptability of Empty-nesters
}

\author{
SHA Kuang-wei
}

(Tianjin Vocational Institute, Tianjin 300410)

\begin{abstract}
Under the background of population aging and social small family structure, empty nests have become a hot topic nowadays, and it is urgent to solve the problem of residential needs of empty nests and the adaptability of elderly living. This paper mainly introduces the summary of empty nests, analyzes the problem of the residential needs of empty nests, explores the measures to improve the adaptability of the elderly living in community, in order to create favorable conditions for empty nesters.
\end{abstract}

Key words: Empty nests, Residential needs, Adaptability.

At present, the increase of empty nests in the cities has become a phenomenon prevailing in the society, to which the reasons are as many to count alongside with significant impact that is mainly centered at the failure to fulfill the residential needs and the adaptability of the empty nesters. To come up with the best workout to the problem of urban empty nests, it is necessary to carry out an in-depth research on the above to explore relevant problems beneath and solutions for improvements with reform measures to implement accordingly.

\section{A General Review on Empty Nests}

Empty nests mainly refers to the families where the elderly are living alone without children, or with their children setting up their own homes, to bear their own for basic necessities of life. From the perspective of family life cycle theory, "empty nest”, beginning with the separation of children and their parents, belongs to the last stage of family development. Currently, with the continuous development of the trend of population aging, the number of urban empty nests has grown gradually, resulting in quite significant social issues, namely the residential needs and adaptability of the elderly that have given rise to concerns by the government and the relevant personnel.

\section{On the Residential Needs of Empty Nests}

The residential needs of empty nests are mainly embodied in physical environment and spiritual contexts. The author will analyzes and studies the problem 
based on the two aspects above.

\subsection{Needs on Physical Environment}

The needs of the empty nesters on the physical environment of the community are shown in the following four aspects, including safety, sites for activities, the comfort and convenience of the surrounding environment, and obstacle-free movement.

First of all, empty nesters may suffer physical damages from fall and the like in life due to physiological factors. In recent years, it is commonly reported for accidents about empty nesters getting hurt as slipping in places like the kitchen or bathroom, which reminds us that it is urgent to improve the living environment for empty nester to provide them with better living quality.

Second, the provision of sites for activities will play an important role in enhancing the mental status of empty nesters as it will gather the majority of empty nesters together who have been long for communicate with people as living alone for a long time. However, not sufficient sites for activities have been set up for empty nesters so far. In this sense, it is necessary to take into action for these sites.

Moreover, the elderly are always in need of a higher degree of comfort and convenience in daily life to facilitate their movements prevented by physiological factors. Therefore, supermarkets and hospitals and other infrastructure are required to be built up in the living environment for the elderly so as to meet the basic needs of empty family in the light of clothing, food, housing, transport and health.

Last but not least, obstacle-free passageways in empty nests should be set to provide the elderly with the maximum accessibility due to their ages and inconvenience in moving and even some are in need of a wheelchair.

\subsection{Needs on Spiritual Contexts}

The main problem of empty nesters comes to a lack of spiritual support in life, which will lead to the loss of pleasure and vitality of the elderly in life and cause to unfavorable physical and physiological changes.

Firstly, a major problem in the living environment of the urban empty nesters is that there is short of recreational facilities and projects provided, which is better in big 
cities while serious in small cities requiring corresponding measures to be taken into effect. With sufficient recreational facilities and entertainment programs, empty nesters are able to maintain physical and mental pleasure, proper social activities and interpersonal relationships, so as to meet their needs in the old years avoiding bad moods e.g. loneliness, negativity and emptiness.

Secondly, it comes to the emotional needs of the empty nesters. For the elderly, it is with companions and lovers by side that they long for. The most the empty nesters yearn for are filial piety, and concerns and cares from spouse, relatives and friends, which will make their lives warm and sweet.

Lastly, the elderly still desire to do something to realize their own value. In this sense, a sense of personal worth also speaks for the residential needs of empty families, which can be fulfilled by various forms such as taking care of their grandchildren, doing housework and participating in social activities and so on, so as to achieve the personal worth mentioned above for the elderly.

\section{Solutions to the Residential Needs of Empty Families to Improve}

\section{Adaptability of the Elderly in the Community}

Based on the analysis above it is obvious that there are problems existing in the residential needs of urban empty nests, which will cause to extremely serious impact on the physical and mental state of empty nesters. Therefore, it is necessary to carry out solutions accordingly and the author suggests as follows:

\subsection{To Improve the Physical Environment of Residential Areas for the Elderly}

First, as for the frequency of getting hurt from slipping of the elderly, it is recommended to have a better workout on the selection of floor materials to enhance skid-resistance in the construction of kitchens and bathrooms, so as to prevent the elderly from risk to the greatest extent. Moreover, the design of the indoor balcony is also very important. For ordinary buildings, the design of the balcony is often based on practical functions, which means less to the elderly. In this regard, the balcony of the residential area for urban empty nester can be designed to connect with the indoor as a whole to maximize the space for moving for the elderly and provide a living 
environment more favorable.

Second, relevant departments should be sufficiently supportive in funding and technical provisions in order to build square and other sites in the neighborhood of the elderly, with facilities and sites provided in the squares in favor of the physical exercises of the elderly to improve their physical health.

Third, basic settings including supermarkets should be provided in the residential areas of the elderly to grant them with the best convenience. At the same time, locate their residential areas close to hospitals as much as possible or establish clinics in their residential areas, so as to make sure that they are able to receive medical treatment timely upon health problems, which does count for great value for the health status of the elderly.

Lastly it comes to the obstacle-free design for the movement of the elderly. It will be best to arrange them living at a lower level, and the power-backup elevator should be provided for higher floors. A gradual slope should adopted to replace a ladder and the bedroom, kitchen, bathroom and other rooms should be set in the same plane as much as possible to facilitate the movement of the elderly, while a sufficient width should be granted for the doorway of the rooms to get a wheelchair through. Public places like elevators, toilets, etc., that the elderly often use should be designed without a threshold, gap or ladder and provided with a slope if necessary. Eye-catching logos should be affixed at inconvenient places such as the start, stop and turn of the stairs and so on to remind the elderly of their speed and change of direction, while obstacle-free design is necessary in the outdoor like using a slope instead of steps, to ensure them the greatest convenience in the outdoor environment.

\subsection{To Improve the Spiritual Contexts of Residential Areas for the Elderly}

It is of great necessity to ensure a good mental state of the elderly for better spiritual life. Corresponding measures should be taken in view of the fact that currently the spiritual contexts in the residential areas for empty nesters are far from perfect.

First, build a cultural activity center around its neighborhood where the elderly enjoy a good environment for culture and art acquisition and make their spiritual life 
rich and fulfilled. At the same time, make the natural scenery indoor and the elderly are able to get spiritual cultivation and satisfaction, which is of significant value to enrich their spiritual contexts.

Second, the loss of their loved ones is the biggest challenge facing the empty nesters that are the most in need of emotional care. Caring services should be organized actively in the residential areas together with a website offering services of warmth and love, and volunteer services should be advocated to spend time with empty nester to bring them attendance and care as their loved ones.

Third, let the elderly feel that they are needed and help them realize their own value. Activities such as community patrols, taking care of the children and construction of the elderly troupe should be held actively in the community, and thereby encourage the elderly to participate in community activities with a sense of social integration to make a contribution of their own for the development of the community.

\subsection{To Establish the System of Residential Adaptability for the Elderly}

In order to address the residential needs of empty nests and improve the adaptability of the elderly living in the community, it requires a sound residential adaptability system for the elderly apart from the complete physical environment and spiritual contexts mentioned above, therefore providing the most comfortable living conditions for the elderly.

First of all, with respect to housing construction, it requires to adjust the system of residential areas to be diversified for the elderly, which consists of empty nest mode and elderly-based mode and the like to meet the contemporary needs of the elderly on different family housing modes. Moreover, establish flexible residential space to adjust the form of housing at any time according to the changes of family members and their opinions to meet the needs varying from family reunion to half-way cohabitation. In addition, renovate the old houses to cater for the needs arousing from the physical and mental changes of the elderly and family structure.

Secondly, as far as the spatial environment is concerned, explore the new principles of living planning to optimize the living environment for the elderly on the 
whole and re-plan all kinds of welfare and service facilities for the elderly. Build a friendly neighborhood and optimize the design of residential units or courtyards to promote close contacts between neighborhoods and enrich the living environment of empty nests, while in the principle of environment construction of maintaining the vitality and independent living of the elderly improving the hardware design of the living environment to meet the physical psychological and social needs of the elderly as far as possible.

Finally, it comes to facilities and services. In the current context of IT application, it has given rise to the needs of the elderly family for the Net of facilities and the diversification of services. In the residential areas, it should carry out planning on the living services and facilities for the elderly in a unified and all-round manner based on the residential adaptability of the elderly, which covers health care, cultural and recreational facilities and household basics etc., to accomplish the services network for the elderly.

\section{Conclusion}

To sum up, the phenomenon of empty nests is widespread in the cities, with a higher degree of requirements imposed on the residential areas. However, the physical environment and spiritual contexts have been inefficient to meet the needs of the elderly and thus lead to serious adverse effects on the physical and mental health of the elderly. Therefore, it is necessary to find solutions to the problems above from the point of view both material and spiritual, so as to meet the needs of empty nester to the greatest extent.

\section{References}

[1]Yuan Deng. On the Construction Research of Housing Modes for Urban Empty Nesters [D]. Central Academy of Fine Arts, 2015 (12) .

[2]Fangbing Wang. On the Research of Needs on the Urban Living Environment for the Elderly [D]. East China Normal University, 2015 (12).

[3] Yuan Zang. On the Research of the Needs of Home Services for Rural Empty

Nesters [D]. Shandong University of Finance and Economics, 2016 (10). 
[4] Jiahao Huang. Research and Reflection on the Needs of Urban Empty NestersTaking Hefei City as an Example [J]. Theoretical Exploration, 2013 (03).

[5] Nan Liu. Study on the Role Adaptation of New "Empty Nest" [D]. East China University of Science and Technology, 2012 (06). 